\title{
Experiments and theory of an upstream ionization instability excited by an accelerated electron beam through a current-free double layer
}

\author{
A. Aanesland, ${ }^{a)}$ M. A. Lieberman, ${ }^{\text {b) }}$ C. Charles, and R. W. Boswell \\ Space Plasma Power and Propulsion, Research School of Physical Sciences and Engineering, \\ The Australian National University, ACT 0200, Australia
}

(Received 5 September 2006; accepted 23 October 2006; published online 6 December 2006)

\begin{abstract}
A low-frequency instability varying from 10 to $20 \mathrm{kHz}$ has been discovered in the presence of a current-free double layer (DL) in a low-pressure expanding helicon plasma. The instability is observed using various electrostatic probes, such as Langmuir probes floating or biased to ion saturation and emissive probes measuring the plasma potential. A retarding field energy analyzer measuring the ion energy distribution function downstream of the double layer is used together with the LP to simultaneously observe the DL and the instability, confirming their coexistence. The frequency of the instability decreases with increasing neutral pressure, increases with increasing magnetic field in the source and increases with increasing rf power. A theory for an upstream ionization instability has been developed, in which electrons accelerated through the DL increase the ionization upstream and are responsible for the observed instability. The theory is in good agreement with the experimental results and shows that the frequency increases with the potential drop of the double layer and with decreasing chamber radius. (c) 2006 American Institute of Physics.
\end{abstract}

[DOI: $10.1063 / 1.2398929]$

\section{INTRODUCTION}

Double layers (DLs) are spatially isolated, rapid changes of the potential in a plasma, and are important structures for accelerating charged particles. They have been invoked in many space and astrophysical plasmas, and are, for example, commonly believed to cause the downward acceleration of the electrons that collide with the upper atmosphere, creating the visible aurora. ${ }^{1}$ Direct observations of localized parallel electric fields in the boundary between the cold dense ionosphere and the hot tenuous magnetospheric plasma have been reported ${ }^{2}$ and support the existence of a large electric double layer at an altitude of about $6000 \mathrm{~km}$ above the visible aurora. Double layers are also thought to accelerate charged particles in solar flares, ${ }^{3}$ and it has recently been suggested that current-free double layers play a significant role in accelerating ions in solar magnetic funnels. ${ }^{4}$

Direct measurements of localized electric fields by spacecrafts and sounding rockets are a challenging task as the spacecrafts have to be at the right place at the right time. The success by the Viking and Freja satellites in measuring "the aurora double layer" have shown that this is possible, but the task becomes even more challenging when considering the solar corona environment and other astrophysical objects. In order to support the theories that double layers exists and play an important role in space and astrophysical plasma environments, "easy" methods to determine the existence of DLs in laboratory plasmas are desirable. A possible method might be provided by the knowledge of the instabilities existing in conjunction with the DL structures.

\footnotetext{
${ }^{a)}$ Electronic mail: aanesland@lptp.polytechnique.fr; new address LPTP, Ecole Polytechnique 91128 Palaiseau cedex, France.

${ }^{b)}$ Permanent address: Dept. EECS-1770, University of California, Berkeley, CA 94720.
}

Recently, a current-free electric double layer (CFDL) that spontaneously forms in expanding low-pressure plasmas was discovered. ${ }^{5,6}$ The spontaneous formation of CFDLs was subsequently confirmed in other experimental systems, ${ }^{7-9}$ and was successfully modeled using particle-in-cell simulations. ${ }^{10}$ The main interest in this paper is to investigate the related instabilities that exist in conjunction with this current-free double layer.

A recently developed theory for the formation of a CFDL incorporates four groups of charges particles: thermal ions, monoenergetic accelerated ions flowing downstream, accelerated electrons flowing upstream, and thermal electrons. ${ }^{11,12}$ The accelerated electrons are formed downstream from a nearly half-Maxwellian electron distribution accelerated through the DL potential drop and enhance the ionization in the upstream high potential region. Measurements of the ion energy distribution functions (IEDFs) using a retarding field energy analyzer (RFEA) have shown the existence of a supersonic ion beam downstream of the DL. ${ }^{6,13}$ Preliminary experimental results have also shown evidence supporting the presence of an electron beam with a velocity that correlates well with the height of the doublelayer potential. ${ }^{6}$

Experiments show that double-layer structures, although generally stable in time, present a high level of fluctuations of different frequencies on both sides of the DL. ${ }^{3}$ At the low-potential side there are two populations of ions that can interact under certain condition and that give rise to a growing low-frequency ion-ion beam instability. ${ }^{14}$ This instability is common in the aurora double-layer environment where ions of different masses drifts at different velocities. ${ }^{15}$ The ion-ion beam instability has been thoroughly investigated in the laboratory and it has been shown, by controlling the ion beam velocity $v_{b}$, that the frequency of the instability is pro- 




FIG. 1. Schematic of "Chi-Kung," a horizontal helicon system. The position of the LP and the RFEA are indicated on the figure.

portional to $v_{b}$ and occurs only for beam velocities smaller than two times the ion acoustic velocity $v_{b}<2 C_{s}{ }^{16,17}$

At the high-potential side of the double layer, the accelerated beam of electrons can cause electron-ion or electronelectron two-stream instabilities. If we keep to the lowfrequency instabilities, the most known examples are the Buneman instability ${ }^{18}$ and the ion-acoustic instability. The plasma is unstable to the Buneman instability if the electron drift velocity exceeds the electron thermal velocity. ${ }^{19}$ An electrostatic ion-cyclotron instability (EICI) can also occur in the upstream region if the electrons are drifting along the magnetic field lines. ${ }^{20}$ The frequency of the EICI depends strongly on the magnetic field and is slightly above the ion gyrofrequency. An ionization instability in the upstream region can also be excited as a consequence of the additional upstream ionization caused by the accelerated electron beam. ${ }^{21}$ The first measurements of low frequency instabilities associated with a CFDL were presented in Ref. 22 and interpreted as an upstream ionization instability. ${ }^{21,23,24} \mathrm{~A}$ theory of the instability was developed based on Refs. 11 and 21 . Both experiment and theory showed that the frequency of the instability increases with the potential drop of the DL.

In this paper, we present experimental results showing the behavior of the ionization instability as a function of pressure, magnetic field, and rf power, and we give a complete description of the ionization instability model. The experimental apparatus and diagnostics are described in Sec. II. In Sec. III, the experimental results of the low-frequency instability are discussed, showing the coexistence of the instability and the CFDL. In Sec. IV, the theory of the upstream ionization instability is presented. The experimental results and the theory are compared in Sec. V, and a conclusion and discussion are given in Sec. VI.

\section{EXPERIMENTAL APPARATUS}

The experiments are performed in the helicon source Chi-Kung, where CFDLs have been previously investigated, ${ }^{5,6}$ and a schematic of the system is shown in Fig. 1. The plasma is created by a helicon-type antenna powered at $13.56 \mathrm{MHz}$, which is wrapped around a cylindrical insulating source chamber. The source is connected to a larger diameter grounded diffusion chamber, and the plasma expands geometrically as well as due to a diverging magnetic field generated by two solenoids placed around the source. In the present study (unless stated otherwise), the magnetic field is about $130 \mathrm{G}$ in the source decreasing to about $10 \mathrm{G}$ in the diffusion chamber (which correspond to $6 \mathrm{~A}$ in each solenoid), the rf power is $250 \mathrm{~W}$ and the pressure is $0.3 \mathrm{mTorr}$.

The CFDL forms spontaneously in the source a short distance from the junction with the diffusion chamber for pressures between 0.2 and 2 mTorr in argon. The upper and lower pressure limits for double-layer formation are discussed thoroughly in Ref. 11. The lower pressure limit is attributed to the ionization rate of accelerated electrons upstream being not sufficient enough to balance the particle loss in the upstream region. The high-pressure limit is reached when the energy relaxation length for ionizing electrons becomes important compared to the system length. It has also been shown previously that a minimum magnetic field of about $120 \mathrm{G}$ is required in the upstream region for the DL to exist in Chi-Kung. ${ }^{25}$

A Langmuir probe (LP) is used to measure the fluctuations of the floating potential $V_{f}$ (probe floating into $1 \mathrm{M} \Omega$ ), the ion saturation current $V_{i, \text { sat }}$ (probe biased at $-56 \mathrm{~V}$ ), and the electron saturation current $V_{e \text {,sat }}$ (probe biased at $+56 \mathrm{~V}$ ). The probe tip is made of a $3 \mathrm{~mm}$ diameter nickel disk, and the probe shaft has a coaxial shielding that terminates $2 \mathrm{~cm}$ from the probe tip. An emissive probe (EP) is used to measure the fluctuations directly on the plasma potential $V_{p}$. The EP is made of a $0.125 \mathrm{~mm}$ thick and $5 \mathrm{~mm}$ long tungsten filament that is heated by $2.4 \mathrm{~A}$. $V_{p}$ is measured directly by floating the probe into $1 \mathrm{M} \Omega{ }^{26}$ An RFEA measuring the IEDF downstream ${ }^{6}$ is used together with the LP to simultaneously observe the presence of the DL (i.e., the presence of a downstream ion beam) and the instability. Additionally the RFEA is used to measure the fluctuations on the beam ion current $I_{\text {beam }}$, directly acquiring the frequency spectrum at chosen, constant discriminator voltages (details are given below).

Data acquisition and fast Fourier transform of the time signal are performed with a National Instruments high-speed digitizer (sampling rate of $100 \mathrm{MHz}$ ) controlled via a LABVIEW diagnostic system. The frequency spectrum is measured over a broad frequency range from $1 \mathrm{kHz}$ to $30 \mathrm{MHz}$. We emphasize here the fluctuations observed at $5-20 \mathrm{kHz}$, which are present only when the CFDL exists. A typical spectrum from 0 to $30 \mathrm{kHz}$ is plotted on Fig. 2, and shows a pronounced peak at $\sim 11 \mathrm{kHz}$. This frequency also appears as sidebands on the $\mathrm{rf}$ driving frequency, 13.56 MHz $\pm(5-20) \mathrm{kHz}$, as shown on the inset in Fig. 2. This coupling between the rf pump wave and the lowfrequency instability is presently being investigated in more detail.

\section{EXPERIMENTAL RESULTS}

The instability frequency $F_{I}$ and amplitude $A_{I}$ have been measured as a function of pressure, magnetic field, rf power, and radial and axial position. The instability is consistently measured on all plasma parameters $\left(V_{f}, I_{i, \text { sat }}, I_{e \text {,sat }}, V_{p}, I_{\text {beam }}\right)$, 


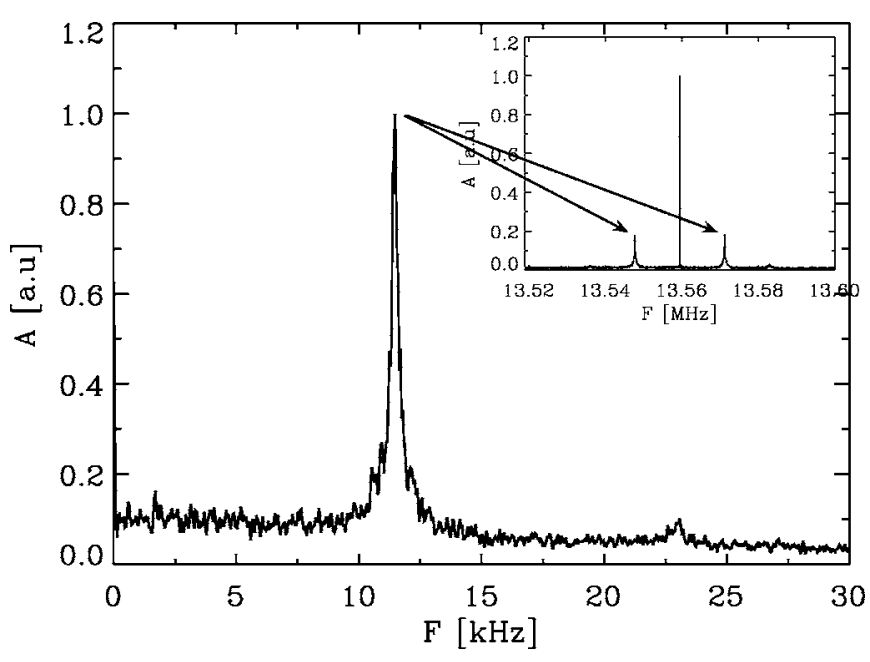

FIG. 2. Typical low-frequency spectrum on the LP when the double layer is present. The pressure is $0.3 \mathrm{mTorr}$, rf power $250 \mathrm{~W}$, the magnetic field is about $130 \mathrm{G}$ in the source and the probe is on-axis about $5 \mathrm{~cm}$ upstream of the DL. The inset shows the corresponding sidebands on the rf driving frequency.

but for clarity only the measurements of $V_{f}$ and $I_{\text {beam }}$ are presented in this paper.

Figure 3 shows the measured frequency of the instability $F_{I}$ and potential drop of the double layer $V_{s}$ as a function of pressure, which shows a linear dependency between $F_{I}$ and $V_{s}{ }^{22}$ The corresponding instability amplitude is plotted on the inset in Fig. 3 and has a maximum at 0.3-0.4 mTorr. The same variation of frequency with pressure has been measured on- and off-axis, upstream and downstream of the DL, with the LP floating or biased to ion saturation, and with the emissive probe. An initial parametric study shows that the instability amplitude is maximum in the upstream region on the $z$ axis. The floating LP is therefore placed on-axis about $5 \mathrm{~cm}$ upstream $(r=0 \mathrm{~cm}, z=20 \mathrm{~cm})$ to ensure the best signal-tonoise ratio, while the RFEA is about $12 \mathrm{~cm}$ downstream of the DL $(r=0 \mathrm{~cm}, z=37 \mathrm{~cm})$ where a thorough study of the



FIG. 3. Frequency of instability (diamonds) and potential drop of the double layer (triangles) as a function of pressure. The instability is measured on the floating LP about $5 \mathrm{~cm}$ upstream of the DL, while the potential drop is measured by the RFEA $12 \mathrm{~cm}$ downstream of the DL. Inset is the normalized amplitude of the instability (data points, and fitted line). Same experimental conditions as for Fig. 2.

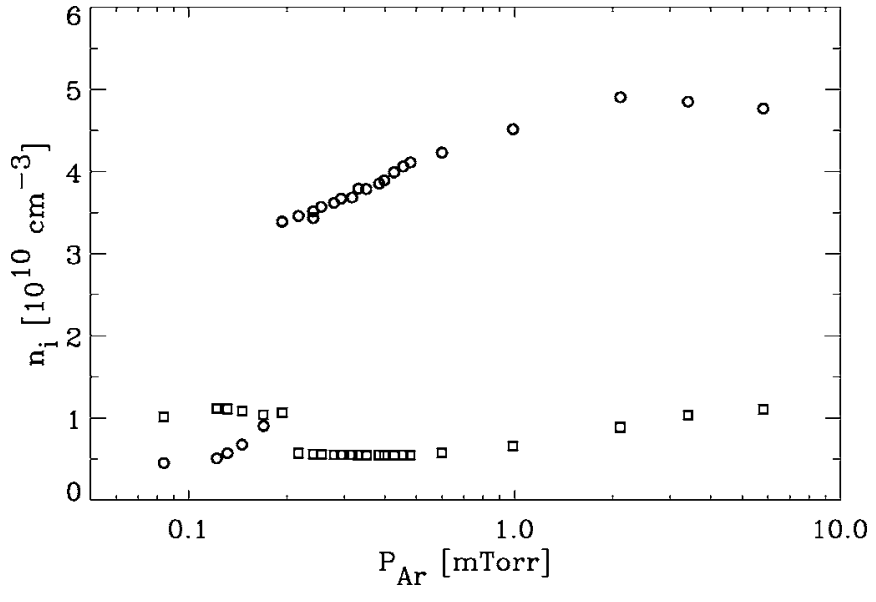

FIG. 4. Plasma density as a function of pressure. Circles and squares are measured $5 \mathrm{~cm}$ upstream and $12 \mathrm{~cm}$ downstream of the DL, respectively, which correspond to the position of the LP and the RFEA in Fig. 3. Same experimental conditions as given in Fig. 2.

DL was obtained previously; the positions of the LP and the RFEA are indicated on Fig. 1.

Simultaneous measurements with the LP and the RFEA confirm that the ion beam and the instability disappear at the same lower pressure threshold of $0.2 \mathrm{mTorr}$. The noise in the low-frequency spectrum increases and the instability amplitude decreases at pressures between 0.6 and $1 \mathrm{mTorr}$, and the frequency could therefore not be measured accurately. However, we observed that the frequency seems to saturate at about $5 \mathrm{kHz}$ before it disappears at $2 \mathrm{mTorr}$. The data points are not plotted at these pressures, as the amplitudes are small and almost "disappear" in the noise.

Figure 4 shows the plasma density as a function of pressure at the upstream and downstream position corresponding to the LP and the RFEA positions in Fig. 3 and indicated on Fig. 1. The plasma density upstream of the DL increases slowly as a function of increasing pressure in the range between 0.2 and 1 mTorr. Hence, the instability amplitude shown on the inset in Fig. 3 does not directly follow the plasma density evolution as a function of pressure. However, the axial and radial variation in $A_{I}$ roughly follow the axial and radial variation in the density when no other parameters are changed. It is evident from these density measurements that there is a mode change at $0.2 \mathrm{mTorr}$ (at the lower pressure threshold for which the DL exists). Below 0.2 mTorr, the density drops significantly and the density in the downstream region exceeds the source density.

Figure 5 shows $F_{I}$ and $V_{s}$ as a function of current in the two source solenoids (i.e., a function of the magnetic field) at a pressure of $0.3 \mathrm{mTorr}$ and with $250 \mathrm{~W}$ rf power. The same current is used in both coils and $6 \mathrm{~A}$ correspond to a maximum magnetic field in the source of $130 \mathrm{G}$, while 3 A gives a magnetic field of $75 \mathrm{G}$ in the source. The downstream magnetic field is roughly $10 \mathrm{G}$ in all cases. The frequency of the instability and the DL potential drop seem to again follow each other, although not as evident as in Fig. 3. Both the double layer and the instability disappears at a magnetic field close to $120 \mathrm{G}(4.5 \mathrm{~A})$. For a coil current of $4 \mathrm{~A}$, a tail of ions at an energy of $7 \mathrm{~V}$ is measured on the IEDF, suggesting 


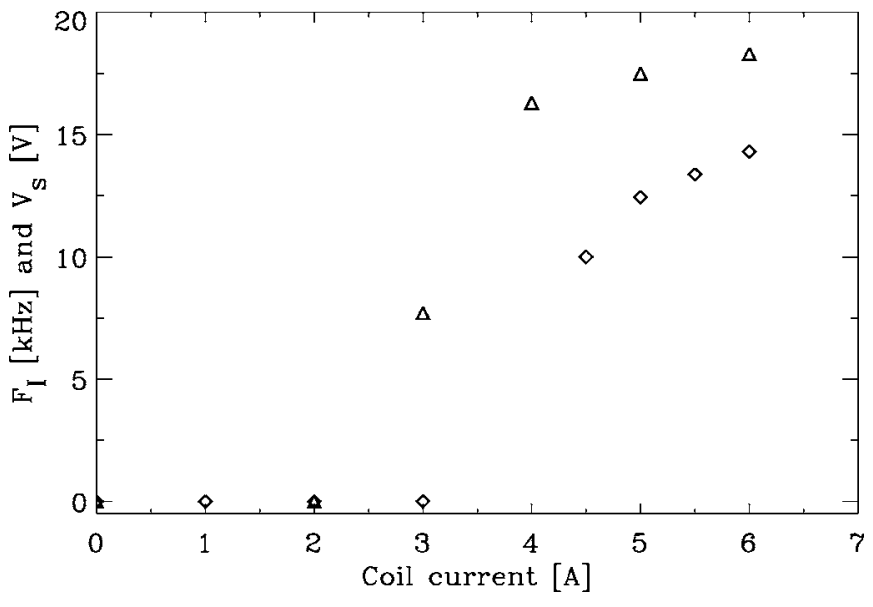

FIG. 5. Frequency of instability (diamonds) and potential drop of the double layer (triangles) as a function of current in both solenoids. The LP and RFEA are at the same positions as for Fig. 3, the rf power is $250 \mathrm{~W}$ and the pressure is $0.3 \mathrm{mTorr}$.

a higher potential in the source but probably no double layer. $^{25}$

Figure 6 shows $F_{I}$ and $V_{s}$ as a function of $\mathrm{rf}$ power. In this case $F_{I}$ increases while $V_{s}$ decreases slowly with increasing $\mathrm{rf}$ power. The instability is observed at an $\mathrm{rf}$ power of $100 \mathrm{~W}$ where the ion beam is not detected at the present RFEA position. The instability could not be detected for $\mathrm{rf}$ powers above $900 \mathrm{~W}$ due to increased noise in the spectrum. The above results will be discussed in Sec. V.

In addition to confirming the coexistence of the DL and the instability, the RFEA is used to study the instability directly on the beam ions. The RFEA characteristic is found by measuring the collector current $I_{c}$ as a function of the discriminator voltage $V_{d}$, where

$$
I_{c}\left(V_{d}\right) \approx \int_{V_{d 0}}^{\infty} v_{\|} f\left(V_{d}\right) d V_{d},
$$

where $f$ is the ion energy distribution function (IEDF) and $v_{\|}$ is the ion velocity parallel to the axis of the analyzer. ${ }^{6}$ Hence,

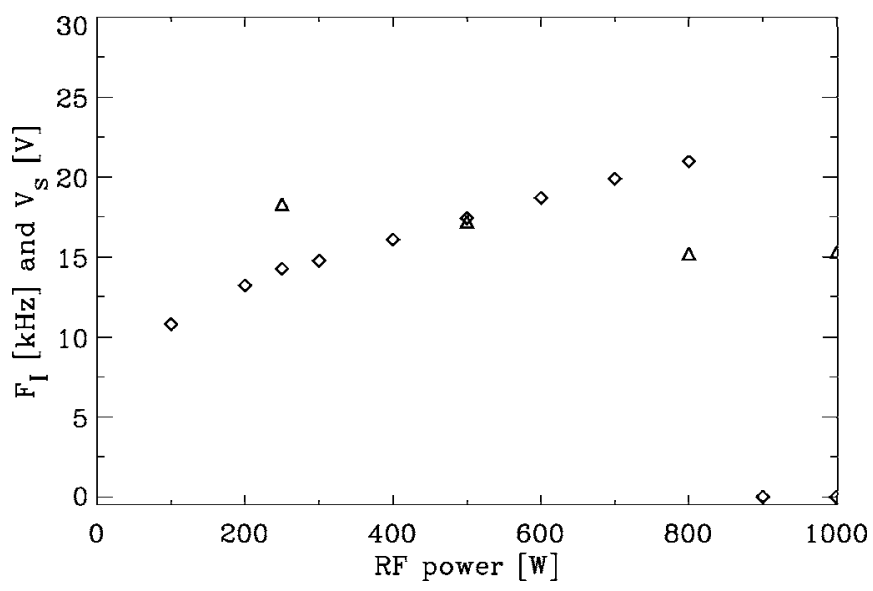

FIG. 6. Frequency of instability (diamonds) and potential drop of the double layer (triangles) as a function of rf power. The LP and RFEA are at the same positions as for Fig. 3, the pressure is $0.3 \mathrm{mTorr}$ and the solenoid current is $6 \mathrm{~A}$.



FIG. 7. Normalized ion energy distribution function (data points and solid line), and the normalized amplitude of the $15 \mathrm{kHz}$ instability measured on the raw RFEA data for successive discriminator voltages (diamonds and dashed-dotted line). The pressure is $0.3 \mathrm{mTorr}$ and the rf power $250 \mathrm{~W}$. (Reprinted with permission from Ref. 22 with copyright 2006 by the American Physical Society).

the IEDF is proportional to the derivative of the collector current. Figure 7 shows the normalized IEDF, i.e., $d I_{c} / d V_{d}$, at a pressure of 0.3 mTorr (data points and fitted solid curve). The IEDF consist of a background cold ion population with an energy corresponding to the plasma potential of $26 \mathrm{~V}$ and an ion beam at an energy peak of $53 \mathrm{~V}$. The ion beam density is about $10 \%$ of the background ions, found by integrating the IEDF from 47 to $60 \mathrm{~V}$. The frequency spectrum is measured on the collector current $I_{c}$ for successive values of discriminator voltages $V_{d}$ and the corresponding amplitude of the instability $A_{I}$ is shown by diamonds on Fig. 7 , the dashed-dotted line being a guide for the eye. Note that the frequency of the instability remains constant at about $15 \mathrm{kHz}$. Interestingly, $A_{I}$ decreases by only $50 \%$ when $V_{d}$ is increased from 0 to $47 \mathrm{~V}$, and then remains fairly constant until no ions can enter the RFEA at $60 \mathrm{~V}$. As discussed above, at a voltage of $47 \mathrm{~V}$, only $10 \%$ of the ions can reach the collector. Rotating the RFEA perpendicular to the ion beam allows one to measure the population of cold background ions only as the directed beam ions cannot enter. ${ }^{6}$ This was found to represent $90 \%$ of the ions at a discriminator voltage of $0 \mathrm{~V}$; however, in this case $A_{I}$ drops by as much as $70 \%$ compared to the equivalent measurement when the RFEA is facing the DL. Hence, the ion beam downstream is strongly influenced by the instability, and we therefore suspect that the instability is "born" upstream and carried by the accelerated ions downstream.

It has been shown previously that a good proportion of the potential drop of the double layer is transformed into the ion beam formation, and at a potential drop of $25 \mathrm{~V}$ (0.2 mTorr) the beam velocity is $2.1 C_{s}{ }^{6}$ This beam velocity exceeds the threshold for the ion-ion beam instability and can therefore not be a plausible candidate explaining our observations.

\section{THEORY OF IONIZATION INSTABILITY}

Johnson et al. ${ }^{21}$ observed low-frequency instabilities in their double plasma device when current-driven DLs were present, and suggested that electrons energized by the DL 
potential drop may excite an ionization instability in the upstream region. As the ionization collision cross section is a rapidly increasing function of the electron energy (close to the ionization threshold), the beam electrons will add to the ionization in the upstream region. The underlying assumption for this instability to grow is the existence of an electrostatic perturbation in the upstream plasma potential; the ionization rate will then be higher at the wave crest, increasing the amplitude of the potential. In the one-dimensional fluid model of Johnson et al., a monoenergetic beam of electrons with energy slightly higher than the ionization energy was assumed to account for all the ionization in the upstream region, and the ionization by the Maxwellian population of background electrons was neglected. This model was later modified to account for the contribution from the cold electron population in addition to assuming a nonquasineutral perturbation. ${ }^{23}$ The model has also been adapted to dusty plasmas, where negatively charged dust grains have been taken into account. ${ }^{24}$

The theoretical analysis presented here includes an accelerated non-Maxwellian group of electrons upstream, due to the flow of downstream Maxwellian electrons, created by electron-neutral ionization, across the double layer. A twodimensional low-pressure diffusion analysis is used. A brief description of the theory was given in Ref. 22. Here we include more details and a discussion of our model.

The plasma equilibrium, with uniform particle densities in the bulk that drop relatively sharply at the plasma-sheath edge, is found in the upstream region by balancing the creation and loss of ions there, yielding

$$
n_{d} n_{g} K_{\mathrm{iz}}+n_{c} n_{g} K_{\mathrm{izc}}-\nu_{L} n_{i}=0,
$$

where $n_{d}$ is the thermal electron density, $n_{g}$ is the neutral gas density, $K_{\mathrm{iz}}\left(T_{e}\right)$ is the thermal electron-neutral ionization rate coefficient, $n_{c}$ is the accelerated (beam) electron density, $K_{\mathrm{izc}}\left(V_{s}, T_{e}\right)$ is the accelerated electron-neutral ionization rate coefficient

$$
\nu_{L}=\left(\frac{2 h_{l}}{l}+\frac{2 h_{R}}{R}\right) u_{B}
$$

is the upstream particle loss frequency, $n_{i}=n_{d}+n_{c}$ is the ion density, $T_{e}$ is the common thermal electron temperature in the upstream and downstream regions, and $u_{B}=\left(e T_{e} / M\right)^{1 / 2}$ is the Bohm velocity, with $M$ the ion mass. In (3), we assume no reduction in the radial losses due to the upstream magnetic field,

$$
h_{l}=\frac{0.86}{\left(3+l / 2 \lambda_{i}\right)^{1 / 2}} \quad \text { and } \quad h_{r}=\frac{0.8}{\left(4+R / \lambda_{i}\right)^{1 / 2}}
$$

are the axial and radial edge-to-center density ratios determined from low-pressure diffusion theory, ${ }^{11,27}$ with $l$ and $R$ the length and radius of the upstream region, respectively, and $\lambda_{i}$ the (constant) ion-neutral mean free path. The ionization rate coefficient $K_{\mathrm{izc}}$ for the upstream accelerated electrons was calculated in ${ }^{11}$ and depends on both $V_{s}$ and $T_{e}$. The second term in (2) corresponds to the energy-dependent electron-neutral ionization cross section used in Refs. 21 and 23, but accounts for the non-Maxwellian distribution of the

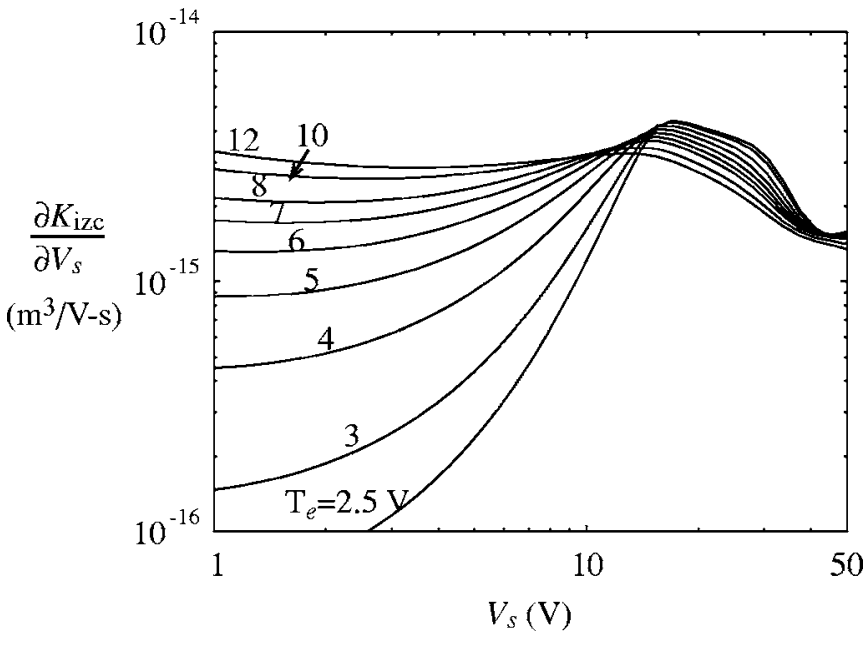

FIG. 8. Derivative of the upstream beam ionization rate coefficient with respect to the double-layer potential drop $K_{\mathrm{izc}}^{\prime}=\partial K_{\mathrm{izc}} / \partial V_{s}$ as a function of $V_{s}$ at various values of $T_{e}$. (Reprinted with permission from Ref. 22 with copyright 2006 by the American Physical Society).

electron beam, rather than assuming a monoenergetic beam. We assume a uniform density for the electron beam everywhere in the upstream region, and the effect of this assumption will be investigated in further work.

Assuming that the perturbed quantities varies as $\exp (j \omega t-j k z)$, the perturbed ion balance/continuity relation is

$$
j \omega \widetilde{n}_{i}+n_{i} \nabla \cdot \widetilde{v}_{i}=\widetilde{n}_{d} n_{g} K_{\mathrm{iz}}+\widetilde{n}_{c} n_{g} K_{\mathrm{izc}}+n_{c} n_{g} K_{\mathrm{izc}}^{\prime} \widetilde{\varphi}-\nu_{L} \widetilde{n}_{i},
$$

where the tilde denotes the perturbed quantities. We have set $\widetilde{K}_{\mathrm{izc}} \simeq K_{\mathrm{izc}}^{\prime} \widetilde{\varphi}$ by a first-order approximation, where the derivative of the accelerated electron ionization rate coefficient with respect to the double-layer potential drop $V_{s}$ is $K_{\mathrm{izc}}^{\prime}$ $=\partial K_{\mathrm{izc}} / \partial V_{s}$, and $\widetilde{\varphi}$ is the perturbed potential in the plasma. $K_{\mathrm{izc}}^{\prime}$ is calculated in Appendix A, and the results for $K_{\mathrm{izc}}^{\prime}$ versus $V_{s}$ are plotted in Fig. 8 for various values of $T_{e}$.

To solve for the wave dispersion, the perturbed quantities $\left(\widetilde{v}_{i}, \widetilde{n}_{d}, \widetilde{n}_{c}\right.$, and $\left.\widetilde{n}_{i}\right)$ are found as a function of $\widetilde{\varphi}$, which allows us to express (5) solely by the perturbed potential. The perturbed ion velocity $\widetilde{v}_{i}$ is found from the first-order ion momentum relation

$$
j \omega M \widetilde{v}_{i}=-e \nabla \widetilde{\varphi}-M \nu_{\mathrm{mi}} \widetilde{v}_{i},
$$

yielding

$$
\widetilde{v}_{i}=-\frac{e}{M} \frac{1}{j \omega+\nu_{\mathrm{mi}}} \nabla \tilde{\varphi}
$$

where $\nu_{\mathrm{mi}}=u_{i} / \lambda_{i}$ is the ion-neutral momentum transfer frequency, with $u_{i}$ the mean ion speed. For $R \ll l$ in the lowpressure regime $T_{i} / T_{e} \ll \lambda_{i} / R \ll 1, u_{i}$ can be well approximated as $u_{i} \approx u_{B} h_{R}$, such that

$$
\nu_{\mathrm{mi}} \approx h_{R} u_{B} / \lambda_{i},
$$

as discussed in Appendix B. 
The linear relation between the thermal electron perturbed density and the perturbed potential is found from the Boltzmann relation

$$
\widetilde{n}_{d}=n_{d}\left(e^{\tilde{\varphi} / T_{e}}-1\right) \simeq n_{d} \frac{\widetilde{\varphi}}{T_{e}},
$$

where the latter equality follows because $\widetilde{\varphi} \ll T_{e}$. Since the upstream accelerated electrons have a non-Maxwellian (beam-like) axial velocity distribution given by (A1), we can use the Vlasov equation

$$
j \omega \tilde{f}_{c}-j k_{z} v_{z} \tilde{f}_{c}-j k_{z} \frac{e}{m} \frac{\partial f_{c}}{\partial v_{z}} \widetilde{\varphi}=0,
$$

to determine the corresponding relation between $\widetilde{n}_{c}$ and $\widetilde{\varphi}$. Solving for the perturbed distribution function $\tilde{f}_{c}$ yields

$$
\tilde{f}_{c}=\frac{e}{m} \frac{k_{z}}{\omega-k_{z} v_{z}} \frac{\partial f_{c}}{\partial v_{z}} \widetilde{\varphi}
$$

Integrating $\widetilde{f}_{c}$ over velocity space determines the perturbed density

$$
\begin{aligned}
\tilde{n}_{c} & =-\frac{e}{m} \widetilde{\varphi} \int_{v_{s}}^{\infty} \frac{1}{v_{z}-\omega / k_{z}} \frac{\partial f_{c}}{\partial v_{z}} d v_{z} \\
& =-\frac{e}{m} \widetilde{\varphi}\left[\int_{v_{s}}^{\infty} \frac{f_{c}}{\left(v_{z}-\omega / k_{z}\right)^{2}} d v_{z}-\frac{f_{c}\left(v_{s}\right)}{v_{s}-\omega / k_{z}}\right],
\end{aligned}
$$

with $v_{s}=\left(2 e V_{s} / m\right)^{1 / 2}$. Although (12) could be evaluated numerically, we use the conditions $\omega / k_{z} \ll v_{s}$ and $T_{e} \ll V_{s}$, which are well -satisfied for the instability regime, to evaluate the integral, yielding

$$
\tilde{n}_{c}=-n_{c} \frac{\widetilde{\varphi}}{2 V_{s}}
$$

This is equivalent to assuming that the accelerated electron flux is conserved:

$$
\Gamma_{c}=\left(n_{c}+\tilde{n}_{c}\right)\left(V_{s}+\widetilde{\varphi}\right)^{1 / 2}=\text { const },
$$

which yields (13) from the linear terms. Note that the signs in (9) and (13) are different, expressing the fact that thermal electrons gather into regions of higher potential, whereas the beam electron density decreases when the velocity increases, as the beam enters a region of higher potential.

We assume a quasineutral perturbation $\widetilde{n}_{i}=\widetilde{n}_{d}+\widetilde{n}_{c}$, where $\widetilde{n}_{d}$ and $\widetilde{n}_{c}$ are given by (9) and (13), respectively, yielding

$$
\widetilde{n}_{i}=\left(\frac{n_{d}}{T_{e}}-\frac{n_{c}}{2 V_{s}}\right) \widetilde{\varphi}
$$

Nonquasineutral perturbations were examined in Refs. 23 and yield corrections of order $\lambda_{\mathrm{D}}^{2} / R^{2} \ll 1$, where $\lambda_{\mathrm{D}}$ is the Debye length.

Finally, substituting all the perturbed quantities into (5) gives the perturbed potential

$$
\begin{aligned}
j \omega( & \left.\frac{n_{d}}{T_{e}}-\frac{n_{c}}{2 V_{s}}\right) \tilde{\varphi}-n_{i}\left(\frac{e}{M} \frac{1}{j \omega+\nu_{\mathrm{mi}}}\right) \nabla^{2} \widetilde{\varphi} \\
& =n_{d} \nu_{\mathrm{iz}} \frac{\widetilde{\varphi}}{T_{e}}-n_{c} \nu_{\mathrm{izc}} \frac{\widetilde{\varphi}}{2 V_{s}}+n_{c} \Delta \nu_{\mathrm{izc}} \frac{\widetilde{\varphi}}{T_{e}}-\nu_{L}\left(\frac{n_{d}}{T_{e}}-\frac{n_{c}}{2 V_{s}}\right) \widetilde{\varphi},
\end{aligned}
$$

where $\nu_{\mathrm{iz}}=n_{g} K_{\mathrm{iz}}, \nu_{\mathrm{izc}}=n_{g} K_{\mathrm{izc}}$, and $\Delta \nu_{\mathrm{izc}}=n_{g} K_{\mathrm{izc}}^{\prime} T_{e}$. The thermal electron ionization frequency $\nu_{\text {iz }}$ was determined in Ref. 11 to be consistent with the "downstream" particle loss rate

$$
\nu_{\mathrm{iz}}=\left(\frac{h_{l_{1}}}{l_{1}}+\frac{2 h_{R_{1}}}{R_{1}}\right) u_{B},
$$

with $h_{l_{1}}=0.86 /\left(3+l_{1} / \lambda_{i}\right)^{1 / 2}$ and $h_{R_{1}}=0.8 /\left(4+R_{1} / \lambda_{i}\right)^{1 / 2}$ the downstream axial and radial edge-to-center density ratios, and $l_{1}$ and $R_{1}$ the downstream length and radius, respectively.

Introducing the upstream accelerated electron fraction $\eta$, such that $n_{c}=\eta n_{i}$ and $n_{d}=(1-\eta) n_{i}$, and introducing the ratio $\epsilon=T_{e} / 2 V_{s}$, then (16) can be written in the form of a Helmholtz equation

$$
\nabla^{2} \widetilde{\varphi}+k^{2} \widetilde{\varphi}=0,
$$

with the wavenumber $k$ given by

$$
\begin{aligned}
k^{2} u_{B}^{2}= & \left(j \omega+\nu_{\mathrm{mi}}\right)\left[(1+\epsilon)(1-\eta)\left(\nu_{\mathrm{iz}}-\nu_{L}\right)+\eta \Delta \nu_{\mathrm{izc}}\right. \\
& -j \omega(1-\eta-\epsilon \eta)],
\end{aligned}
$$

where $\nu_{\mathrm{izc}}$ is eliminated using (2). Equation (19) is a quadratic equation for $\omega$ of the form

$$
A \omega^{2}+j B \omega-C=0,
$$

with the coefficients

$$
\begin{aligned}
& A=1-\eta-\epsilon \eta, \\
& B=\eta \Delta \nu_{\mathrm{izc}}-\nu_{\mathrm{mi}}(1-\eta-\epsilon \eta)-(1-\eta)(1+\epsilon)\left(\nu_{L}-\nu_{\mathrm{iz}}\right),
\end{aligned}
$$

$$
C=k^{2} u_{B}^{2}+\nu_{\mathrm{mi}}(1-\eta)(1+\epsilon)\left(\nu_{L}-\nu_{\mathrm{iz}}\right)-\nu_{\mathrm{mi}} \eta \Delta \nu_{\mathrm{izc}} .
$$

Solving (20) yields the wave frequency for any given (real) wavenumber $k$. For $4 A C-B^{2}>0$, the solution for $\omega$ has real and imaginary parts given by

$$
\operatorname{Re} \omega=\frac{\left(4 A C-B^{2}\right)^{1 / 2}}{2 A} \text { and } \operatorname{Im} \omega=-\frac{B}{2 A} .
$$

The wave is unstable for $\operatorname{Im} \omega<0$; i.e., $B>0$. For $4 A C$ $-B^{2}<0$, the wave has Re $\omega=0$ and is unstable for $B>0$.

The above derivation applies when there is an accelerated group of electrons (accelerated by $V_{s}$ ) that can add to the ionization. In the case when $V_{s}=0$, there is no double layer in the plasma and no electron beam $\left(n_{c}=0\right)$. In this case the upstream plasma equilibrium is

$$
n_{i} n_{g} K_{\mathrm{iz}}-\nu_{L} n_{i}=0,
$$

yielding $\nu_{\mathrm{iz}}=\nu_{L}$. The perturbed ion continuity equation is then 
TABLE I. Corresponding values used for the five pressure cases plotted on Figs. 9-11. $V_{s}$ is found experimentally using Fig. $3, T_{e}$ is calculated using (17), and $K_{\mathrm{izc}}^{\prime}$ is found numerically using Fig. 8.

\begin{tabular}{cccccc}
\hline \hline Case & $P_{\mathrm{Ar}}(\mathrm{mTorr})$ & $V_{s}(\mathrm{~V})$ & $T_{e}(\mathrm{eV})$ & $K_{\mathrm{izc}}^{\prime}\left(\mathrm{m}^{3} / \mathrm{V} \mathrm{s}\right)$ & Observations \\
\hline $\mathbf{a}$ & 0.1 & 0 & 11.8 & $18.9 \times 10^{-15}$ & No DL \\
$\mathbf{b}$ & 0.2 & 23.0 & 6.1 & $3.22 \times 10^{-15}$ & DL case \\
$\mathbf{c}$ & 0.5 & 14.2 & 4.5 & $3.89 \times 10^{-15}$ & DL case \\
$\mathbf{d}$ & 1.0 & 8.5 & 3.7 & $1.58 \times 10^{-15}$ & DL case \\
$\mathbf{e}$ & 3.0 & 0 & 2.9 & $0.38 \times 10^{-15}$ & No DL \\
\hline \hline
\end{tabular}

$$
j \omega \widetilde{n}_{i}+n_{i} \nabla \cdot \widetilde{v}_{i}=\widetilde{n}_{i} n_{g} K_{\mathrm{iz}}-\nu_{L} \tilde{n}_{i} .
$$

Solving for this set of equations in a similar manner as above gives $A=1, B=-\nu_{\mathrm{mi}}$, and $C=k^{2} u_{B}^{2}$, which is equivalent to (21) $-(23)$ when $\eta=0$ and $\epsilon=0$. This solution represents a damped ion acoustic mode in the upstream region with $\operatorname{Re} \omega=\frac{1}{2}\left(4 k^{2} u_{B}^{2}-\nu_{\mathrm{mi}}^{2}\right)^{1 / 2}$ and $\operatorname{Im} \omega=\nu_{\mathrm{mi}} / 2>0$.

The dispersion relation $\operatorname{Re} \omega(k)$ in (24) is calculated for different experimental conditions using the coefficients $A, B$, and $C$. Note that we use $F=\operatorname{Re} \omega / 2 \pi$ in the following calculations. To compare the theory with the measurements, we use the experimental values $l=31 \mathrm{~cm}, R=6.85 \mathrm{~cm}$, $l_{1}=29.4 \mathrm{~cm}$, and $R_{1}=15.9 \mathrm{~cm}$, which gives $h_{l}=0.43$, $h_{R}=0.38, h_{l_{1}}=0.39$, and $h_{R_{1}}=0.36$. The variables in (21)-(23) are functions of the neutral pressure $P_{\mathrm{Ar}}$, the double-layer potential drop $V_{s}$, and the electron temperature $T_{e}$. In order to calculate the dispersion relation "solely" as a function of pressure, a set of $V_{s}$ and $T_{e}$ is found for each pressure; $V_{s}$ is obtained experimentally using Fig. 3 , and $T_{e}$ is calculated theoretically using (17). We find numerically the values of $K_{\text {izc }}^{\prime}$ (Fig. 8) for the corresponding sets of $\left(V_{s}, T_{e}\right)$. Table I shows the potential drop, temperature and differential rate coefficient $\left(K_{\mathrm{izc}}^{\prime}\right)$ for five different pressures; cases $\mathbf{b}$, c, and $\mathbf{d}$ are DL conditions using $\eta=0.27$ and $\epsilon=T_{e} / 2 V_{s}$, and cases $\mathbf{a}$ and $\mathbf{e}$ are non-DL conditions where $\eta=0$ and $\epsilon=0$. The dispersion relations for these five pressure sets are shown on Fig. 9. No propagating mode exists for very low- $k$ numbers, as found also in Ref. 21; however, in our situation there is no upper limit, as the viscosity term used in Ref. 21 is considered negligible here. From an experimental point of view, the physical dimensions of the experiment will introduce some minimum/maximum allowed wavenumbers, as is discussed in more detail below. Im $\omega$ given by (24) is independent of $k$ and is negative for the DL conditions, allowing the instability to grow.

The ratio $\eta$ of beam electron to plasma density was found in Ref. 11 and is a weak function of both $V_{s}$ and $T_{e}$. For simplicity, we used an average density ratio $\eta=0.27$ in the above calculation. To investigate the influence of $\eta$, the imaginary part of the dispersion relation (24) is calculated as a function of $\eta$ for the three DL pressure conditions given by cases $\mathbf{b}-\mathbf{d}$ in Table I. As shown in Fig. 10 there is a threshold density ratio for which the instability changes from a damped to an unstable mode, which is $\eta=0.25,0.14$, and 0.22 for $0.2,0.5$, and 1.0 mTorr, respectively. Hence, although the accelerated electron beam always adds to the ion-

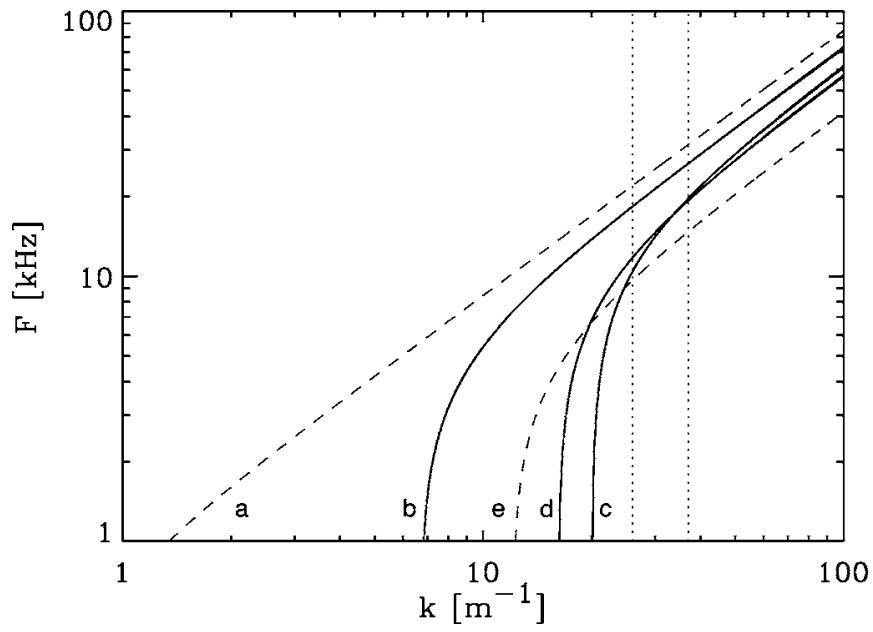

FIG. 9. The dispersion relation $F(k)$ given by Eq. (24), where $F=\operatorname{Re} \omega / 2 \pi$, is plotted for five pressures: 0.1, 0.2, 0.5, 1.0, and $3.0 \mathrm{mTorr}$ with indexes $\mathbf{a}, \mathbf{b}, \mathbf{c}, \mathbf{d}$, and $\mathbf{e}$, respectively. The corresponding set of $\left(V_{s}, T_{e}, K_{\mathrm{izc}}^{\prime}\right)$ are detailed in Table I. The solid lines correspond to DL conditions where the growth rate $\operatorname{Im} \omega<0$, and the dashed lines are non-DL conditions where $\operatorname{Im} \omega>0$. The vertical dotted lines indicate $k=26 \mathrm{~m}^{-1}$ and $k=37 \mathrm{~m}^{-1}$.

ization, the model predicts that the amplification or growth of the initial fluctuation requires a minimum electron beam density.

Figure 11 shows the calculated dispersion relation for 0.2 mTorr (case b in Table I) where $\eta$ varies from $0.17,0.21$, 0.27 , to 0.37 , and all other parameters are kept constant. The solid lines are unstable solutions with $\operatorname{Im} \omega<0$ and the dashed lines are stable solutions with $\operatorname{Im} \omega>0$. The frequency does not change much with $\eta$ above $k=20 \mathrm{~m}^{-1}$. Using the average value of $\eta=0.27$ in the following calculations will therefore not alter the calculated frequency significantly, as long as $k>20 \mathrm{~m}^{-1}$.



FIG. 10. Growth rate $\operatorname{Im} \omega$ as a function of the density ratio $\eta$ between the beam electrons and the background plasma density for the DL cases $\mathbf{b}$, c, and $\mathbf{d}$ shown on Fig. 9 and with details given in Table I. The vertical dotted line indicates $\eta=0.27$. 


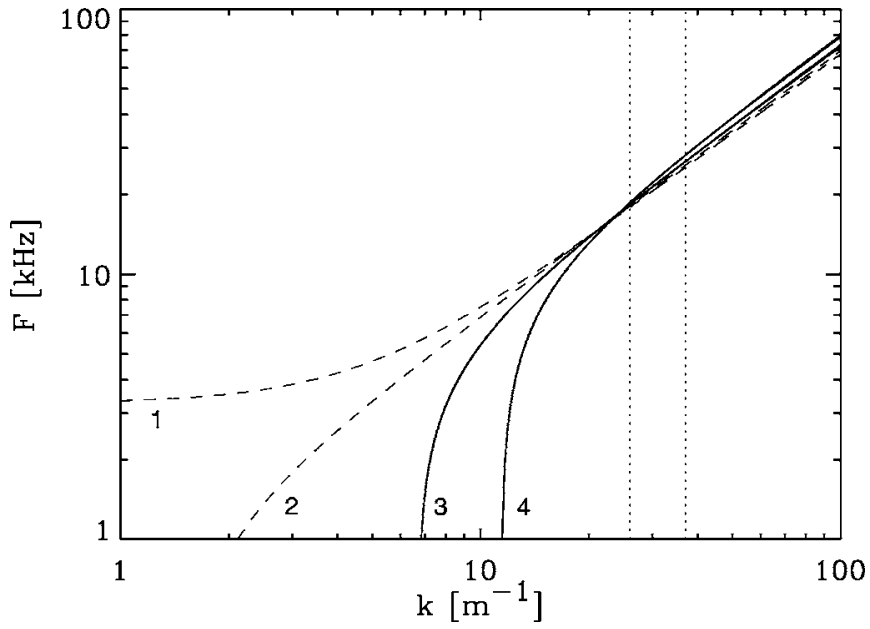

FIG. 11. Dispersion relation for 0.2 mTorr (index b) where indexes 1, 2, 3, and 4 are calculated using $\eta=0.17,0.21,0.27$, and 0.37 , respectively, with all other parameters constant. The solid lines are unstable modes and the dashed lines are damped modes. The vertical dotted lines indicate $k=26 \mathrm{~m}^{-1}$ and $k=37 \mathrm{~m}^{-1}$, as for Fig. 9 .

\section{COMPARING EXPERIMENTS AND THEORY}

\section{A. Instability frequency versus pressure}

To fully compare the theory with the experimental results presented in Sec. III, the frequency of the instability is calculated as a function of pressure keeping the wavenumber $k$ constant. The calculation is performed using the above method, finding a set of $\left(V_{s}, T_{e}, K_{\mathrm{izc}}^{\prime}\right)$ for a range of pressures between 0.2 and 1 mTorr. Figure 12 shows the calculated (solid line) and measured (diamonds) frequency as a function of pressure for $k=26 \mathrm{~m}^{-1}$. There is good agreement between the calculated and measured frequency. The calculated frequency increases slightly in the pressure range where the low-frequency noise in the spectrum was too high for the instability to be detected experimentally (0.6-2 mTorr). The growth rate, here represented by the coefficient $B$ in (24) and shown by the dotted line, is positive, although very small in this pressure regime.

We have used a wavenumber $k=26 \mathrm{~m}^{-1}$ in the calculation as this fits very well to the experimental results. However, $k$ can be estimated theoretically in (18) by centering a cylindrical coordinate system on-axis at the midplane of the upstream region, which gives $\widetilde{\varphi}=\widetilde{\varphi}_{0} \cos k_{z} z J_{0}\left(k_{r} r\right)$ with $k_{z}^{2}+k_{r}^{2}=k^{2}$. Making a plausible assumption for the boundary conditions that $\tilde{\varphi}=0$ at $z= \pm l / 2$ and $\tilde{\varphi}=0$ at $r=R$, we obtain $k_{z}$ and $k_{r}$ for the normal mode with the smallest eigenvalue $k^{2}$ given by $k_{z}=\pi / l$ and $k_{r}=\chi_{01} / R$, with $\chi_{01} \approx 2.405$ the first zero of the zero-order Bessel function $J_{0}$. From this we obtain $k=37 \mathrm{~m}^{-1}$. The narrow spectra presented in Fig. 2 suggest that there is a standing wave component that needs a reflection mechanism, in agreement with the above assumption.

The wavenumber is determined mainly by the radial variation of the perturbed potential and density given by $k_{r}$. In order to justify a somewhat reduced wavenumber $k_{r}, \widetilde{\varphi}$,

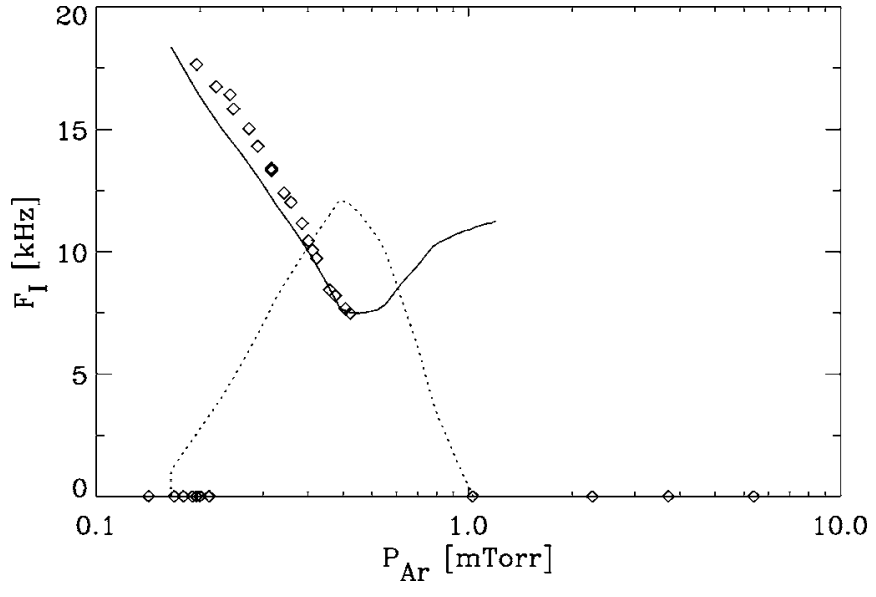

FIG. 12. Calculated (solid line) and measured (diamonds) frequency of the instability as a function of pressure. The diamonds are replotted from Fig. 3. The calculation is performed with $k=26 \mathrm{~m}^{-1}$ and $\eta=0.27$. The dotted line is the "normalized" $B$ coefficient $\left[B\left(2.5 \times 10^{-4}\right)\right]$ showing an unstable mode.

and $\tilde{n}$ might not fall to zero at the chamber radius, but at some larger effective radius. Using $r=10 \mathrm{~cm}$ rather than $6.8 \mathrm{~cm}$ gives $k=26 \mathrm{~m}^{-1}$, as used in Fig. 12 .

\section{B. Magnetic field and power dependence}

In the above calculation, we found a set of $V_{s}, T_{e}$, and $K_{\mathrm{izc}}^{\prime}$, corresponding to a certain pressure, which allowed us to calculate the frequency "solely" as a function of pressure. We now want to calculate the frequency as a function of magnetic field in the same manner to compare with the experimental result shown on Fig. 5. In this case, the pressure is constant at 0.3 mTorr, and since the temperature is mainly a function of pressure, we assume $T_{e}$ to be constant, equal to $4 \mathrm{eV}$. For $p=0.3 \mathrm{mTorr}$ and $T_{e}=4 \mathrm{eV}$, we find $\nu_{\mathrm{mi}}=1.1$ $\times 10^{4} \mathrm{~s}^{-1}, \nu_{L}=4.1 \times 10^{4} \mathrm{~s}^{-1}$, and $\nu_{\mathrm{iz}}=1.7 \times 10^{4} \mathrm{~s}^{-1}$, independent of the magnetic field. With the small variation in $V_{s}$ from 15 to $18 \mathrm{~V}$ (Fig. 5), it is well justified that $K_{\mathrm{izc}}^{\prime} \approx 3.5$ $\times 10^{-15} \mathrm{~m}^{3} / \mathrm{V} \mathrm{s}$ for $4-\mathrm{eV}$ electrons (Fig. 8), which gives $\Delta \nu_{\mathrm{izc}}=1.4 \times 10^{5} \mathrm{~s}^{-1}$. Hence, the only parameter that changes as a function of magnetic field in Eq. (24) is the wavenumber $k$. A constant $k=25 \mathrm{~m}^{-1}$ gives a frequency of $14 \mathrm{kHz}$, in agreement with the measurement at $6 \mathrm{~A}$, but fails to explain the decrease in the frequency as a function of decreasing magnetic field. Our model does not directly take into account the influence of the magnetic field; however, when the magnetic field is reduced, the radial boundary condition may change. We already showed in the previous $0.2 \mathrm{mTorr}$ calculation that $k=26 \mathrm{~m}^{-1}$ (effective radius of $10 \mathrm{~cm}$ ) fits better to the experimental results than the theoretical boundary condition $k=37 \mathrm{~m}^{-1}(r=6.85 \mathrm{~cm})$. At $0.3 \mathrm{mTorr}$, the frequency evolution in Fig. 5 measured experimentally, where $F_{I} \approx 14 \mathrm{kHz}$ at $6 \mathrm{~A}$ and $11 \mathrm{kHz}$ at $4.5 \mathrm{~A}$, is in agreement with the model if we assume that $k$ goes from 25 to $20 \mathrm{~m}^{-1}$ (an increase in effective radius) when the solenoid current decreases from 6 to 4.5 A (i.e., the maximum field decreases from 135 to $100 \mathrm{G}$ ).

A similar wavenumber effect is evident in the results of frequency as a function of rf power (Fig. 6). In this case, the density is proportional to the power absorbed, however, the 
DL theory itself ${ }^{11}$ is independent of absolute density; it determines only density ratios. Thus, according to the theory, $\eta$ and $K_{\mathrm{izc}}^{\prime}$ should not change much with power. Hence, all the parameters except for $V_{s}$ and $k$ can also in this case be considered constant as a function of power. If $k$ is constant, Eq. (24) predicts an almost constant frequency as a function of power, which is in contradiction with the measurements shown on Fig. 6. However, the sheath widths and capacitive coupling across the sheath to the radial wall might vary with power (i.e., density), so when the power increases the effective radius decreases and it is likely that $k$ increases. In the pressure range around 0.3 mTorr the frequency is approximately proportional to $k$, as seen in Fig. 9, and is in good agreement with the measured results shown on Fig. 6 .

\section{CONCLUSION AND DISCUSSION}

In summary, we have investigated experimentally a lowfrequency instability occurring in low-pressure plasmas created in an insulated, helicon-driven source chamber connected to a larger diameter, grounded diffusion chamber. The instability exists only when a current-free double layer exists at the junction of the two chambers. The instability is observed for beam velocities exceeding the threshold for the ion-ion beam instability. The experimental results are in good agreement with a theory of an ionization instability that we have developed, in which an electron beam accelerated by the potential drop of the DL adds to the ionization in the upstream source region. The frequency of the instability increases as a function of increasing double-layer potential drop and with increasing wavenumber.

The low-frequency instability has also been observed as sidebands on the helicon source driving frequency 13.56 MHz $\pm(5-20) \mathrm{kHz}$. There are also some instabilities observed in the 120 and $250 \mathrm{kHz}$ range localized in the diffusion chamber at the edge of the ion beam and which also seem to exist in conjunction with the double layer. The $\mathrm{GHz}$ range has not yet been investigated in this experimental system. The ionization instability has also been observed in xenon plasma when the DL is present, and the results are in agreement with Ref. 21. The xenon instability has a frequency slightly lower than in the argon case and is $8 \mathrm{kHz}$ at 0.26 mTorr. The xenon DL exists for lower pressures than in argon and the frequency decreases from 10 to $7 \mathrm{kHz}$ when the pressure increases from 0.17 to 0.44 mTorr.

The ionization instability is shown to exist in conjunction with electron beams that are energetic enough to add to the ionization in the wave crest of an initial potential oscillation. Observation of this instability can be used both in laboratory and space environments to indicate the existence of a double layer and its strength.

\section{ACKNOWLEDGMENTS}

We are grateful for the expert technical assistance by P. Alexander, and for the help from C. Constanzo in performing the xenon experiments. A.A. also acknowledges fruitful discussions with R. Schrittwieser and A. Fredriksen on currentdriven double-layer instabilities.

\section{APPENDIX A: DERIVATIVE OF IONIZATION RATE COEFFICIENT $K_{\mathrm{izc}}^{\prime}$ FOR ACCELERATED ELECTRONS}

To determine $K_{\mathrm{izc}}^{\prime}$, we modify the procedure to determine the upstream ionization rate coefficient $K_{\mathrm{izc}}$ for accelerated electrons given in Ref. 11, in which $K_{\mathrm{izc}}$ was found by integrating $\sigma_{\mathrm{iz}} v f_{c}$ over spherical coordinates in velocity space. Here, $\sigma_{\text {iz }}$ is the measured argon ionization cross section, $v$ is the electron speed, and

$$
\begin{aligned}
& f_{c}=\frac{n_{c}}{\operatorname{erfc}\left(\zeta_{s}\right)}\left(\frac{m}{2 \pi e T_{e}}\right)^{3 / 2} \mathrm{e}^{-m v^{2} / 2 e T_{e}} ; \\
& \left|v_{z}\right|>v_{s}=0, \quad \text { otherwise, }
\end{aligned}
$$

is the velocity distribution, with erfc the complementary error function, and $\zeta_{s}=\left(V_{s} / T_{e}\right)^{1 / 2}$. In Ref. 11 the ionization rate coefficient was found to be

$$
K_{\mathrm{izc}}=\left(\frac{8 e T_{e}}{\pi m}\right)^{1 / 2} \frac{1}{T_{e}^{2} \operatorname{erfc}\left(\zeta_{s}\right)} \mathcal{I}\left(V_{s}, T_{e}\right)
$$

where

$$
\mathcal{I}=\int_{V_{s}}^{\infty} d \mathcal{E} \mathcal{E}\left[1-\left(\frac{V_{s}}{\mathcal{E}}\right)^{1 / 2}\right] \sigma_{\mathrm{iz}}(\mathcal{E}) \mathrm{e}^{-\mathcal{E} / T_{e}} .
$$

Differentiating (A2) with respect to $V_{s}$, we obtain the derivative

$$
K_{\mathrm{izc}}^{\prime}=\frac{\partial K_{\mathrm{izc}}}{\partial V_{s}}=K_{\mathrm{izc}}\left[\frac{1}{\left(\pi T_{e} V_{s}\right)^{1 / 2} \operatorname{erfc}\left(\zeta_{s}\right)} \mathrm{e}^{-V_{s} / T_{e}}-\frac{1}{2} \frac{\mathcal{J}}{\mathcal{I}}\right],
$$

with

$$
\mathcal{J}=\int_{V_{s}}^{\infty} d \mathcal{E}\left(\frac{\mathcal{E}}{V_{s}}\right)^{1 / 2} \sigma_{\mathrm{iz}}(\mathcal{E}) \mathrm{e}^{-\mathcal{E} / T_{e}} .
$$

The numerical results for $K_{\mathrm{izc}}^{\prime}$ versus $V_{s}$ are plotted in Fig. 8 for various values of $T_{e}$.

\section{APPENDIX B: ION-NEUTRAL MOMENTUM TRANSFER FREQUENCY}

The ion-neutral momentum transfer frequency can be written as

$$
\nu_{\mathrm{mi}}=n_{g} \sigma_{\mathrm{mi}} u_{i},
$$

with $u_{i}$ the mean ion speed. For $R \ll l$ in the pressure regime, $T_{i} / T_{e} \ll \lambda_{i} / R \ll 1$, the speed is the radial ion drift velocity

$$
u_{i}=\mu_{i} E,
$$

where $\mu_{i}$ is ion mobility and $E$ is the radial ambipolar electric field. An estimate for the low-pressure mobility is 27

$$
\mu_{i}=\left(2 e \lambda_{i} / \pi M E\right)^{1 / 2},
$$

with $\lambda_{i}=1 / n_{g} \sigma_{\mathrm{mi}}$ the (constant) ion-neutral mean free path. The electric field is estimated as

$$
E=-T_{e}\left(\frac{1}{n} \frac{d n}{d r}\right) \approx \frac{T_{e}}{R} .
$$

Substituting (B3) and (B4) into (B2) yields 


$$
u_{i} \approx 0.8 u_{B}\left(\lambda_{i} / R\right)^{1 / 2} \text {. }
$$

An extension of (B5) to the collisionless (Langmuir) pressure regime $\lambda_{i} \gg R$ is obtained using the edge-to-center density ratio $h_{R}$, through the substitution in (B5) that

$$
0.8\left(\lambda_{i} / R\right)^{1 / 2}-\rightarrow h_{R},
$$

with $h_{R}$ given in (4). Using (B6) to evaluate (B1) then yields

$$
\nu_{\mathrm{mi}} \approx h_{R} u_{B} / \lambda_{i}
$$

${ }^{1}$ H. Alfven, Tellus 10, 104 (1958).

${ }^{2}$ R. Ergun, Y.-J. Su, L. Andersson, C. Carlson, J. McFadden, F. Mozer, D. Newman, M. Goldman, and R. Strangeway, Phys. Rev. Lett. 87, 045003 (2001).

${ }^{3}$ M. Raadu, Phys. Rep. 178, 25 (1989).

${ }^{4}$ R. W. Boswell, E. Marsch, and C. Charles, Astrophys. J. 640, L199 (2006).

${ }^{5}$ C. Charles and R. Boswell, Appl. Phys. Lett. 82, 1356 (2003).

${ }^{6}$ C. Charles and R. Boswell, Phys. Plasmas 11, 1706 (2004).

${ }^{7}$ S. A. Cohen, N. Siefert, S. Stange, E. Scime, and F. Levinton, Phys. Plasmas 10, 2593 (2003).

${ }^{8}$ X. Sun, A. M. Keesee, C. Biloiu, E. E. Scime, A. Meige, C. Charles, and R. W. Boswell, Phys. Rev. Lett. 95, 025004 (2005).

${ }^{9}$ O. Sutherland, C. Charles, N. Plihon, and R. W. Boswell, Phys. Rev. Lett. 95, 205002 (2005).
${ }^{10}$ A. Meige, R. Boswell, C. Charles, and M. Turner, Phys. Plasmas 12, 052317 (2005)

${ }^{11}$ M. A. Lieberman, C. Charles, and R. W. Boswell, J. Phys. D 39, 3294 (2006).

${ }^{12}$ M. A. Lieberman and C. Charles, Phys. Rev. Lett. 97, 045003 (2006).

${ }^{13}$ C. Biloiu, X. Sun, E. Choueiri, F. Doss, E. Scime, J. Heard, R. Spektor, and D. Ventura, Plasma Sources Sci. Technol. 14, 766 (2005).

${ }^{14}$ V. Lapuerta and E. Ahedo, Phys. Plasmas 9, 3236 (2002).

${ }^{15}$ J.-E. Wahlund, F. R. E. Forme, H. J. Opgenoorth, M. A. L. Persson, E. V. Mishin, and A. S. Volokitin, Geophys. Res. Lett. 19, 1919 (1992).

${ }^{16}$ N. Hershkowitz, T. Romesser, G. Knorr, and C. K. Goertz, Phys. Rev. Lett. 33, 754 (1974).

${ }^{17}$ T. Fujita, T. Ohnuma, and S. Adachi, Plasma Physics 19, 875 (1977).

${ }^{18}$ O. Buneman, Phys. Rev. 115, 503 (1959).

${ }^{19}$ J. DeGroot, C. Barnes, A. Walstead, and O. Buneman, Phys. Rev. Lett. 38, 1283 (1977).

${ }^{20}$ J. J. Rasmussen and R. W. Schrittwieser, IEEE Trans. Plasma Sci. 19, 457 (1991).

${ }^{21}$ J. C. Johnson, N. D’Angelo, and R. L. Merlino, J. Phys. D 23, 682 (1990).

${ }^{22}$ A. Aanesland, C. Charles, M. A. Lieberman, and R. W. Boswell, Phys. Rev. Lett. 97, 075003 (2006).

${ }^{23}$ L. Conde, Phys. Plasmas 11, 1955 (2004).

${ }^{24}$ N. D’Angelo, Phys. Plasmas 4, 3422 (1997).

${ }^{25}$ C. Charles, Phys. Plasmas 12, 044508 (2005).

${ }^{26}$ J. R. Smith, N. Hershkowitz, and P. Coakley, Rev. Sci. Instrum. 50, 210 (1979).

${ }^{27}$ M. Lieberman and A. Lichtenberg, Principles of Plasma Discharges and Materials Processing, 2nd ed. (Wiley-Interscience, New York, 2005). 